\title{
Computational and Physical Phantom Setups for the Second cardiac Motion Analysis Challenge (cMAC2)
}

\author{
M. De Craene ${ }^{1}$, P. Allain ${ }^{1}$, H. Gao ${ }^{4}$, A. Prakosa ${ }^{5}$, S. Marchesseau ${ }^{5}$, \\ O. Somphone ${ }^{1}$, L. Hilpert ${ }^{2}$, A. Manrique ${ }^{3}$, H. Delingette ${ }^{5}$, S. Makram-Ebeid ${ }^{1}$, \\ N. Villain ${ }^{1}$, J. D'hooge ${ }^{4}$, M. Sermesant ${ }^{5}$, and E. Saloux ${ }^{2}$ \\ ${ }^{1}$ Philips Research Medisys, France, \\ 2 Department of Cardiology, University Hospital of Caen, Caen, France, \\ ${ }^{3}$ Department of Human Investigations, GIP Cyceron, Caen, France, \\ ${ }^{4}$ Laboratory of Cardiovascular Imaging and Dynamics, University of Leuven, \\ Leuven, Belgium, \\ ${ }^{5}$ Inria, Asclepios Research Project, Sophia Antipolis, France.
}

\begin{abstract}
This paper describes the data setup of the second cardiac Motion Analysis Challenge $(\mathrm{cMac} 2)$. The purpose of this challenge is to initiate a public data repository for the benchmark of motion and strain quantification algorithms on 3D ultrasound images. The data currently includes synthetic images that combine ultrasound and biomechanical simulators. We also collected sonomicrometry curves and ultrasound images acquired on a Polyvinyl alcohol phantom.
\end{abstract}

\section{Introduction}

Being a wide-spread modality, echocardiography plays a key role in the assessment of cardiac function. When it comes to quantifying local motion and strain, the validation of Doppler-based or speckle-tracking measurements becomes critical. Accuracy, sensitivity and reproducibility of any quantification algorithm should be known as a function of the signal to noise ratio before applying it for diagnostic purposes. However, the construction of a ground truth for motion and strain is a challenging task. Indeed, obtaining ground truth from manual measurements requires to track anatomical landmarks over time. Taking the spatial derivative of these trajectories to compute strain amplifies intra- and inter-observer errors.

\subsection{Existing validation strategies}

Several alternatives have been proposed to construct ground truth for motion and strain quantification from ultrasound (US) images.

Another modality can be taken as reference (e.g. tagged Magnetic Resonance (MR) [1]). While this approach can enhance consistency between the two modalities, differences could raise from some bias introduced by the reference modality. 


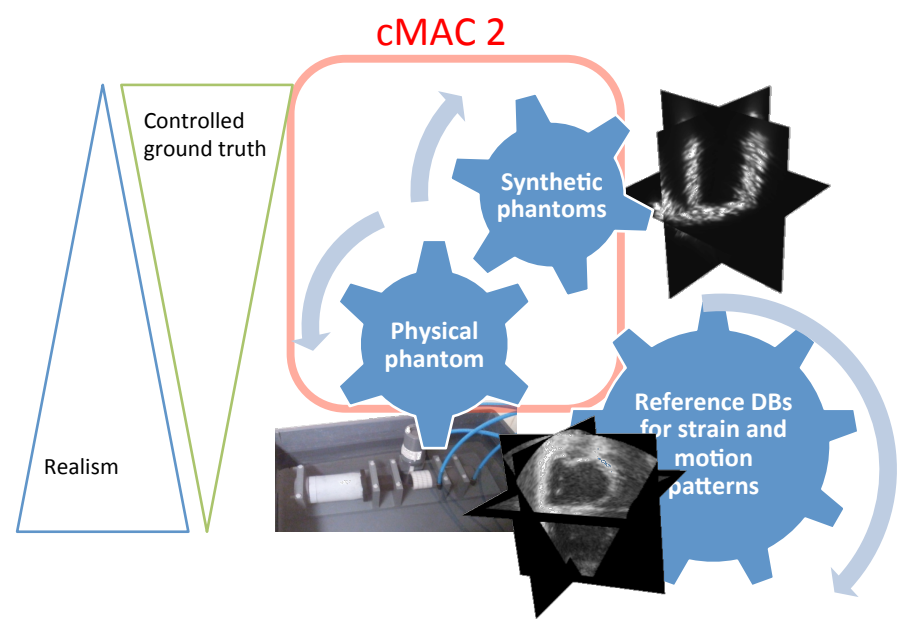

Fig. 1. Global picture of ultrasound-based motion and deformation validation. Covering the whole spectrum between data realism and controlled ground truth requires covering, on the long term, phantom and patient data. This year cMAC2 challenge will be restricted to synthetic images and in vitro phantom to focus on a reliable ground truth motion field.

Sonomicrometry is an alternative for strain validation [2] and measures at a high frame rate the time taken by an acoustic wave to travel between pairs of crystals. Time measurements can be converted to distances if the propagation velocity of the medium is known. However, this data is only available at few locations in the entire volume.

Computational phantoms. To provide highly controlled ground truth datasets, several authors proposed to simulate the US imaging pipeline. While of limited realism, the advantage is to provide data where the exact underlying motion field is known and controlled. The Field [3] package provides a library for the calculation of pressure fields from arbitrarily shaped, apodized, and excited US transducers. It can generate the spatial impulse response specific to an US system with known characteristics. Recently, Gao et al. [4] proposed a fast approach for generating 3D US sequences in less than an hour. It accelerates the convolution of a 3D point spread function (PSF) by multiple 1D convolutions while preserving the resulting image quality. Elen et al. [5] applied this simulation technology to an ellipsoid model of the left ventricle (LV) with torsion, longitudinal and radial deformation mapped to the characteristics of a healthy subject. For inclusion of more realistic geometries and motion, Duan et al. [6] integrated a realistic electro-mechanical model in the simulation process.

Physical phantoms. Alternatively to simulated data, ground truth can be obtained on physical phantoms, which motion and deformations can be mechan- 
ically controlled [7]. Although the geometry of phantoms are often simplified, the obtained image quality reflects the challenges of a real US imaging system. Polyvinyl alcohol (PVA) gels [8] were proposed as a tissue-mimicking material for MR and US. As the number of freeze-thaw cycles affects the properties of the material, locally stiffer inclusions can be embedded for quantifying the localizability of lesions for several spatial and stiffness extents [9].

\subsection{Standardization context in echocardiography}

Public dissemination of validation data is a key issue. Without systematic data access, reproducibility of the findings of the different motion and strain quantification techniques can be hardly verified by independent experts. Recently, the European Association of Echocardiography (EAE) launched a joint initiative with the American Society of Echocardiography ASE to involve manufacturers and software developers in a programme aimed to standardize quantitation of myocardial deformation (strain) among vendors ${ }^{1}$. In this context, different vendors and academic partners regularly meet at cardiac imaging conferences to agree on standard data and formats. The investigations of the task force are currently limited to 2D US imaging.

\subsection{Contribution of cMAC challenges}

At last year STACOM workshop, a motion challenge was proposed [10], including data collected from healthy volunteers and in vitro phantoms. Magnetic resonance (MR) images were acquired using 3D cine and tagging protocols [10]. 3D US images were also collected for the phantom and the volunteers. Although of high quality and realism, the main difficulty for this dataset was the construction of ground truth data for comparing the different motion algorithms. The retained solution was to manually track tag crossings by two observers. The obtained ground truth trajectories on 8 landmarks were then reported on the US space of coordinates. Because of variations in heart rate between MR and US acquisitions, only the end-systolic and end-diastolic results were compared.

Since the focus of this year cMAC challenge is 3D US, our objective was to propose a 3DUS database with highly reliable ground truth on motion and deformation, without requiring another imaging modality or manual measurements to build the ground truth. Physical and computational phantoms are two elements of a complete validation strategy illustrated in Fig. 1. When adding patient images, the resulting database covers the full spectrum from accurate ground truth to fully realistic data. As a first step towards this goal, we propose in this paper a computational phantom obtained from combining an US imaging modeling package [4] with a bio-mechanical model $[11,12]$. We also designed a physical phantom able of dissociating compression and torsion for separately quantifying the accuracy on these two deformation modes. The phantom is made of PVA gel for further allowing local inclusions with different mechanical properties.

\footnotetext{
${ }^{1}$ http://bit.1y/esc-news
} 


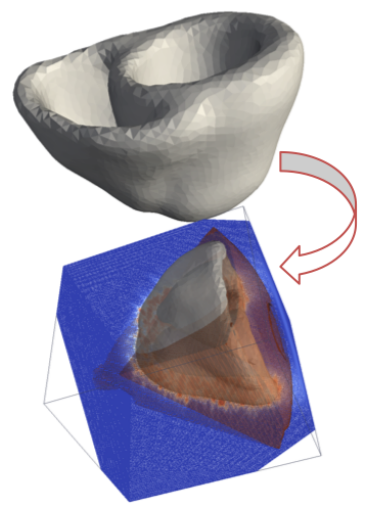

(a)

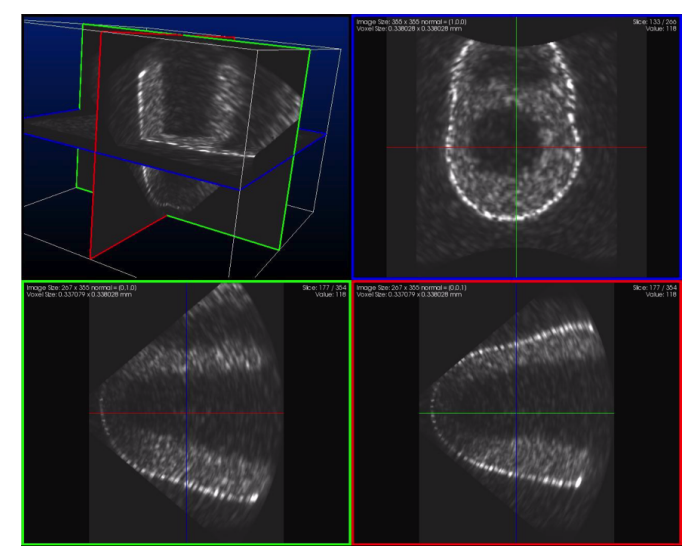

(b)

Fig. 2. Synthetic 3DUS image generation process: (a) Mapping from the simulation volumetric meshes into the 3D US imaging space and (b) obtained image.

\section{Computational phantom}

The synthetic images proposed in this challenge combine an electro-mechanical model described in $[11,12]$ with an US imaging model from [4]. We provide 10 sequences spanning different values of electrical activation, conductivity and contractility parameters. Global conductivity is the conduction velocity of the electrophysiology model and global contractility is the maximum value of the fibre active stress. For electrical delay, both LBBB and RBBB cases were considered with or without pacing in different AHA regions. A summary of the simulation parameters for each case is given in Table 1 . In the current dataset, a single probe design was considered. Scatterers were randomly placed in the myocardial geometry and moved along the cardiac cycle according to the result of the mechanical simulation. A single rigid transformation was used to map the sequence of volumetric meshes to the synthetic US field of view, as illustrated in Fig. 2(a). The US modeling pipeline is fully described in [4].

The 3D Point Spread Function (PSF) considered in the current dataset was obtained by rotating a $2 \mathrm{D}$ beam profile to get $3 \mathrm{D}$ images on the assumption that PSF in azimuth and elevation are the same. Scatterers in the background were placed randomly at each frame to ensure there is no frame to frame correlation in the blood pool. As an additional challenge, scatterers with a high reflectivity were placed around the epicardium to mimic pericardium. This layer moves at a slower rate than the myocardium to render the difficulty brought by an intense pericardium tissue moving at a different speed than the myocardium next to it. An example of 3D US image generated for this challenge is shown in Fig. 2(b). 
Table 1. Simulation parameters for the synthetic database.

\begin{tabular}{|c|l|l|l|}
\hline Case & $\begin{array}{l}\text { Initial Electrical Activation Posi- } \\
\text { tion }\end{array}$ & $\begin{array}{l}\text { Global Con- } \\
\text { ductivity } \\
(\mathrm{cm} / \mathrm{s})\end{array}$ & $\begin{array}{l}\text { Global Con- } \\
\text { tractility } \\
\text { (dimension- } \\
\text { less) }\end{array}$ \\
\hline \hline 1 & Normal & 50 & 0.09 \\
8 & RBBB & 30 & 0.05 \\
12 & LBBB & 30 & 0.05 \\
20 & LBBB+Pacing(AHA 5) & 30 & 0.05 \\
22 & LBBB+Pacing(AHA 6) & 30 & 0.09 \\
28 & LBBB+Pacing(AHA 7) & 30 & 0.05 \\
36 & LBBB+Pacing(AHA 12) & 30 & 0.05 \\
44 & RBBB+Pacing(AHA 9) & 30 & 0.05 \\
60 & BV Pacing(AHA 3+6) & 30 & 0.05 \\
88 & BV Pacing(AHA 14+7) & 30 & 0.05 \\
\hline
\end{tabular}

\section{Physical phantom}

The phantom was made of a single cylindrical cavity. This shape was produced by repeated freezing and thawing of a PVA solution (with addition of graphite). A pneumatic part is mounted at the top and allows varying compression and torsion modes jointly or separately. The mechanical controller is linked to an EEG simulator. The phantom is fully MR compatible. For this challenge, 3D US data was acquired with a Philips 3D X5 probe. In the current setup, data was acquired from a lateral position, as indicated in Fig. 3. Ground truth data for deformation was captured using sonomicrometry. It gives the distance between crystals pairs embedded in the gel and will therefore approximate radial, circumferential and longitudinal strain values. Crystals were placed at a position and using a pattern plotted in Fig. 4. Since the correspondence between image and sonomicrometry coordinate systems is unknown, the four crystals were manually landmarked by an expert and their location in the image was provided to all challengers. The distances between pairs of crystals give an approximation of radial, circumferential and longitudinal strains. Fig. 5 plots the relative length changes over time $\left(\Delta L(t) / L\left(t_{0}\right)\right)$ for the first provided dataset.

\section{Discussion and conclusions}

This paper presented a small database containing both synthetic and phantom datasets. Synthetic images were obtained by combining a biventricular geometry with biomechanical and US imaging models. Although these images do not reproduce faithfully all artifacts inherent to real US images, the true motion field is known densely in the whole myocardial volume. In future work, various settings will be generated for realistic probe designs. The relative intensity of 

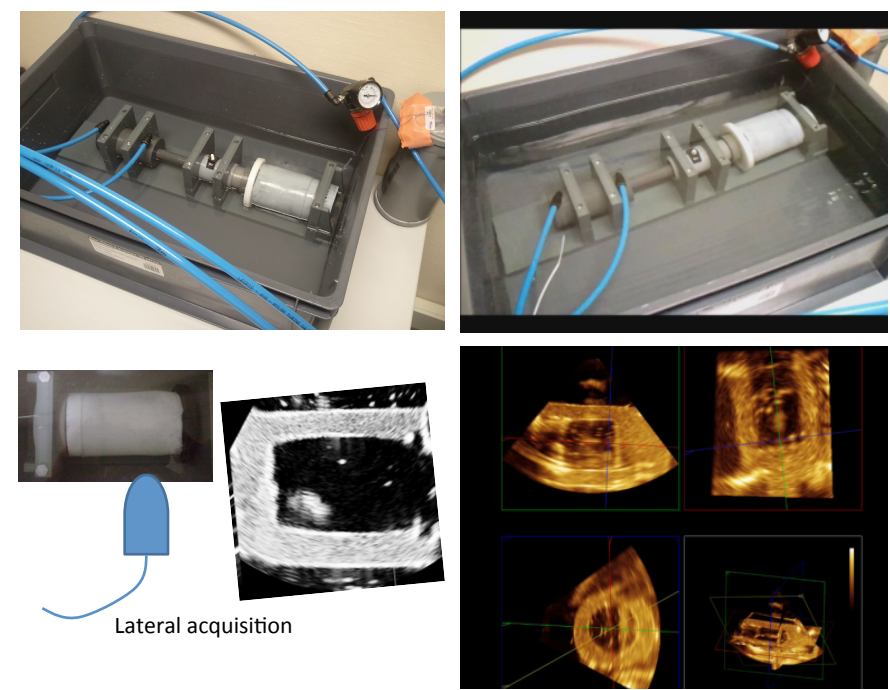

Fig. 3. Physical phantom setup. Top: The PVA phantom is placed in a water-filled aquarium. The pneumatic mechanical part is MR compatible and can dissociate or combine compression and rotation. Bottom left: images were acquired from a lateral position of the probe. Bottom right: obtained 3D US image.

the myocardial wall and the blood pool will be modulated to produce several scenarios of signal to noise ratios.

Regarding mechanical simulations, the current challenge modeled different activation patterns producing normal and impaired motion and deformation. This will help to determine if currently available image processing algorithms can detect and quantify dyssynchrony in the LV. Besides assysnchrony, another pathology we intend to include in the database is the presence of ischemic or stunned segments with reduced or null contractility. These segments should follow the vascularization territories corresponding to the main branches of the coronary tree (left, right and circumflex coronary arteries).

On the physical phantom, ground truth is currently available as pairwise distance curves returned by the sonomicrometry system. This has three main limitations.

First, this information is extremely sparse (4 spatial locations only). To circumvent this, we intend to complement the database by tagged MR acquisitions giving dense markers, the tags crossings, in the myocardium. To this end, we will use the same acquisition protocol as in the first cMAC challenge [10].

Second, sonomicrometry provides ground truth on deformation and not on trajectories. This would be a desirable feature as verifying the accuracy on the motion field is an intermediate step before quantifying strain accuracy. On the physical phantom, there are two ways to construct ground truth trajectories: 


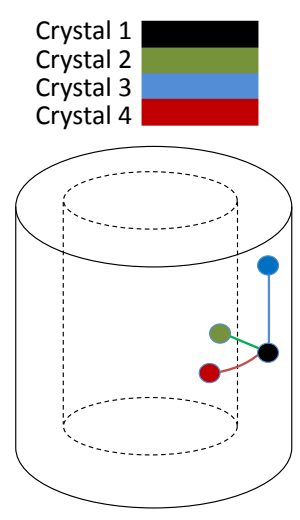

(a)

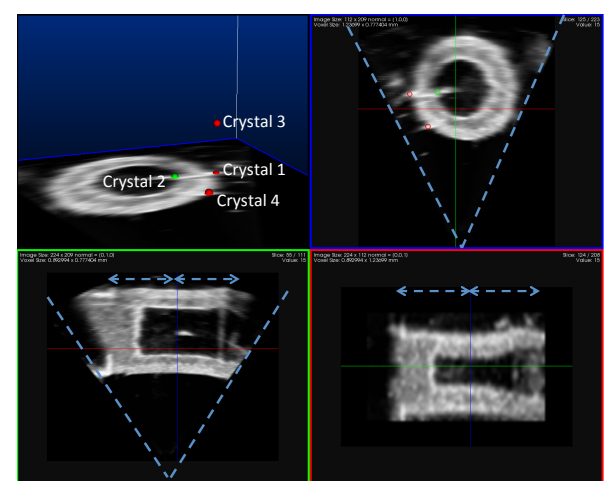

(b)

Fig. 4. Location of the sonomicrometry crystals. (a) Four crystals were placed to capture radial, circumferential and longitudinal length changes. (b) The crystals can be manually landmarked in the image space to compute the same length changes from the intensity-based tracking result. Blue arrows indicate that the crystals are placed at half the height of the cylinder.

one is to manually track tags crossings [10]. An alternative could be to use the sonomicrometry system to reconstruct trajectories by means of triangulation techniques using fixed crystals. This approach was followed by Gorman et al. [13], who applied a multidimensional scaling algorithm for tracking the threedimensional geometry of the mitral valve using sonomicrometry.

Third, sonomicrometry crystals do not give access to the infinitesimal Lagrangian strain tensor, but measure relative length changes approximating strain values. For the "circumferential" direction, microsonometry measures the straight distance between crystals rather than the arc length, as desired for circumferential strain. Despite these limitations, the accuracy of image tracking algorithms can still be quantified if the distances are measured consistently in image and sonomicrometry spaces.

This dataset is only meant as a first step to help the medical imaging processing community to construct reference databases for the validation of motion and strain quantification algorithms from 3D US images. Although embryonic, we hope it will be an opportunity to strengthen a community including physicians and engineers around this challenging topic. All the data generated in this context is hosted on the cardiac atlas project page ${ }^{2}$ and publicly available through email request to help constructing an open validation framework for US-based $3 \mathrm{D}$ strain measurements.

\footnotetext{
${ }^{2}$ http://www . cardiacatlas.org/
} 


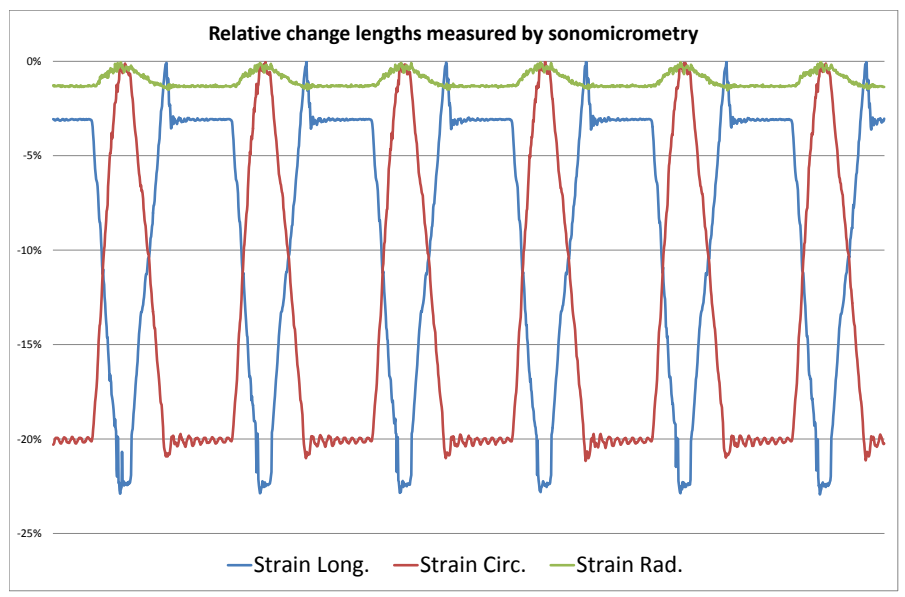

Fig. 5. Pairwise distances between crystals as provided by sonomicrometry.

\section{References}

1. Amundsen, B., Crosby, J., Steen, P., Torp, H., Slørdahl, S., Støylen, A.: Regional myocardial long-axis strain and strain rate measured by different tissue doppler and speckle tracking echocardiography methods: a comparison with tagged magnetic resonance imaging. European Journal of Echocardiography 10(2) (2009) 229-237

2. Amundsen, B., Helle-Valle, T., Edvardsen, T., Torp, H., Crosby, J., Lyseggen, E., Støylen, A., Ihlen, H., Lima, J., Smiseth, O., et al.: Noninvasive myocardial strain measurement by speckle tracking echocardiography: validation against sonomicrometry and tagged magnetic resonance imaging. Journal of the American College of Cardiology 47(4) (2006) 789-793

3. Jensen, J.: A new calculation procedure for spatial impulse responses in ultrasound. The Journal of the Acoustical Society of America 105(6) (1999) 3266-3274

4. Gao, H., Choi, H., Claus, P., Boonen, S., Jaecques, S., Van Lenthe, G., Van Der Perre, G., Lauriks, W., D'hooge, J.: A fast convolution-based methodology to simulate 2-D/3-D cardiac ultrasound images. IEEE Transactions on Ultrasonics, Ferroelectrics and Frequency Control 56(2) (2009) 404-409

5. Elen, A., Choi, H., Loeckx, D., Gao, H., Claus, P., Suetens, P., Maes, F., D'hooge, J.: Three-dimensional cardiac strain estimation using spatio-temporal elastic registration of ultrasound images: A feasibility study. IEEE Transactions on Medical Imaging 27(11) (2008) 1580-1591

6. Duan, Q., Moireau, P., Angelini, E., Chapelle, D., Laine, A.: Simulation of 3D ultrasound with a realistic electro-mechanical model of the heart. Functional Imaging and Modeling of the Heart (2007) 463-473

7. Lesniak-Plewinska, B., Cygan, S., Kaluzynski, K., D’hooge, J., Zmigrodzki, J., Kowalik, E., Kordybach, M., Kowalski, M.: A dual-chamber, thick-walled car- 
diac phantom for use in cardiac motion and deformation imaging by ultrasound. Ultrasound in Medicine \& Biology 36(7) (2010) 1145-1156

8. Surry, K., Austin, H., Fenster, A., Peters, T.: Poly (vinyl alcohol) cryogel phantoms for use in ultrasound and MR imaging. Physics in Medicine and Biology 49 (2004) 5529

9. Heyde, B., Cygan, S., Choi, H., Lesniak-Plewinska, B., Barbosa, D., Elen, A., Claus, P., Loeckx, D., Kaluzynski, K., D'hooge, J.: Regional cardiac motion and strain estimation in three-dimensional echocardiography: A validation study in thick-walled univentricular phantoms. IEEE Transactions on Ultrasonics, Ferroelectrics and Frequency Control (2011) 668-682

10. Tobon-Gomez, C., De Craene, M., Dahl, A., Kapetanakis, S., Carr-White, G., Lutz, A., Rasche, V., Etyngier, P., Kozerke, S., Schaeffter, T., et al.: A multimodal database for the 1st cardiac motion analysis challenge. Statistical Atlases and Computational Models of the Heart. Imaging and Modelling Challenges (2012) 33-44

11. Sermesant, M., Chabiniok, R., Chinchapatnam, P., Mansi, T., Billet, F., Moireau, P., Peyrat, J., Wong, K., Relan, J., Rhode, K., et al.: Patient-specific electromechanical models of the heart for the prediction of pacing acute effects in CRT: A preliminary clinical validation. Medical Image Analysis (2011) 201-215

12. Marchesseau, S., Delingette, H., Sermesant, M., Rhode, K., Duckett, S., Rinaldi, C., Razavi, R., Ayache, N.: Cardiac mechanical parameter calibration based on the unscented transform. Medical Image Computing and Computer Assisted Intervention (2012) Accepted to appear.

13. Gorman III, J., Gupta, K., Streicher, J., Gorman, R., Jackson, B., Ratcliffe, M., Bogen, D., Edmunds, L., et al.: Dynamic three-dimensional imaging of the mitral valve and left ventricle by rapid sonomicrometry array localization. The Journal of thoracic and cardiovascular surgery 112(3) (1996) 712-724 